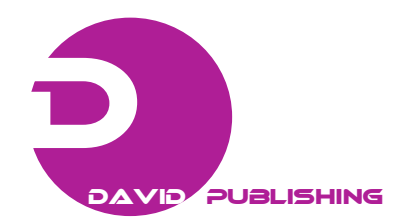

\title{
More on Fatigue Verification of Class 1 Nuclear Power Piping According to ASME BPV Code
}

\author{
Lingfu Zeng ${ }^{1}$ and Lennart G. Jansson ${ }^{2}$ \\ 1. Section for Nuclear Technology, AF-Industry AB, Göteborg 401 51, Sweden \\ 2. Section for Technical Analysis, AF-Industry AB, Göteborg 401 51, Sweden
}

Received: January 24, 2014 / Accepted: March 04, 2014 / Published: June 30, 2014.

\begin{abstract}
Fatigue verification of Class 1 nuclear power piping according to ASME Boiler and Pressure Vessel Code, Section III, NB-3600, which is often discussed in connection to power uprate and life-extension of aging reactors in recent years, is dealt with. Key parameters involved in the fatigue verification, e.g., the alternating stress intensity $S_{a l t}$, the penalty factor $K_{e}$ and the cumulative damage factor $U$, and relevant computational procedures applicable for the assessment of low-cycle fatigue failure using strain-controlled data, are particularly addressed. A so-called simplified elastic-plastic discontinuity analysis for alternative verification when fatigue requirements found unsatisfactory, and the procedures provided in NB-3600 for evaluating the alternating stress intensity $S_{a l t}$, are reviewed in detail. An in-depth discussion is given to alternative procedures suggested earlier by the authors using nonlinear finite element analyses, which uses a nonlinear finite element analysis for directly determining the alternating stress, thus eliminating uncertainties resulted from the use of the penalty factor $K_{e}$. Using this alternative, unavoidable plastic strains can be correctly taken into account in a computationally affordable way, and the reliability of the verification will not be affected by uncertainties introduced in the simplified elastic-plastic analysis.
\end{abstract}

Key words: Piping, fatigue, ASME BPV code, finite element, cyclic plasticity.

\section{Introduction}

A nuclear piping system which is found to be disqualified, i.e., overstressed, in design evaluation using linear analysis software e.g., PIPESTRESS [1] in accordance with ASME Boiler \& Pressure Vessel Code, Section III [2] can still be qualified if further design requirements can be satisfied in refined non-linear finite element analyses in which material plasticity and other non-linear conditions are taken into account. For convenience and clarity, a design evaluation using such linear analysis software will throughout this paper be called a linear design evaluation, and a design evaluation using non-linear finite element analyses a non-linear design evaluation.

Corresponding author: Lingfu Zeng, Ph.D., Mem. ASME, research fields: pressure vessel and piping, nuclear technology, computational solid \& structural mechanics, finite element methods. E-mail: Lingfu.zeng@afconsult.com.
In recent years, many non-linear analyses and verifications have been conducted in Sweden. Unfortunately, most of such work has always ended with, or can never be ended without, long discussions on unclear and inconsistent issues appearing in the ASME Boiler \& Pressure Vessel Code, Section III, which will be denoted as ASME BPV III or simply the code below.

To clarify the design requirements and non-linear finite element analysis procedures that are required, we have in our earlier work attempted to categorize the design rules in ASME BPV III into linear design and non-linear design rules and examined, in particular, those non-linear design rules [3-5]. The ultimate goal of the work was to overcome several difficulties that we have experienced when non-linear design rules applied in connection to our on-going power uprate projects for several BWR reactors in Sweden. 
One difficulty is, among several others that we have experienced while conducting the power uprate projects, the fatigue verification required for Class 1 piping. According to ASME BPV III, NB-3600, the verification shall be made by first using a purely linear elastic analysis, and if an unsatisfactory result predicted, a procedure using so-called simplified elastic-plastic discontinuity analysis should be used as an alternative. Two questions arisen naturally are: (1) How good is the so-called simplified elastic-plastic discontinuity analyses; (2) What other alternatives, which are legal or allowed in accordance with NB-3600, can be used for a further fatigue verification when simplified elastic-plastic discontinuity analyses found to be unsatisfactory. Zeng et al. [6] addressed their general answers to these questions on the basis of their experience from the power uprate projects. In this paper, a more in-depth discussion is given. Our emphasis is again placed on other non-linear finite element procedures alternative to the simplified elastic-plastic discontinuity analysis. We aim at establishing an alternative approach such that unavoidable plastic strains can be correctly taken into account in a computationally affordable way, and the reliability of the verification will not be affected by uncertainties introduced in the simplified elastic-plastic analysis.

The paper is organized as follows: In Section 2, serving as an introduction of various terminologies, an overview of the general linear design principle is given. In Section 3, we describe loads and load combinations which are to be considered and, in particular, we clarify the definition of reversing and non-reversing dynamic loads, with which various design requirements are closely associated. In Section 4, we first review and discuss the linear fatigue verification, and then the alternative verification using a so-called simplified elastic-plastic discontinuity analysis. In Section 5, we address the central issue of this paper-An alternative non-linear design verification procedure. Finally, in Section 6 , concluding remarks are given.
As the paper covers a large amount of design rules and requirements of ASME BPV III, an attempt has been made in order to keep the presentation brief and concise, yet still sufficiently clear. Our principal goal is to clarify those non-linear design rules and their relevant non-linear finite element analysis procedures. Hence, the review of the linear design verification will be kept brief whenever possible. Furthermore, fundamental design requirements, e.g., Pressure Design ,etc., will not be discussed here. This paper is one of a series of our papers which attempt to clarify and examine the design rules and requirements in ASME BPV III in order to form a correct and practically applicable methodology, based on which a nuclear piping system can be correctly analyzed [3-6].

\section{General Linear Design Principle}

The linear design rules according to ASME BPV III are purely based on stress limits. Stresses in piping components are first divided into membrane, bending and localized stresses. Thereafter, stresses are further categorized into primary, secondary and peak stresses. The primary stresses are important to avoid catastrophic failure and to control plastic deformation. The secondary stresses are responsible for an eventual progressive/incremental deformation. The peak stresses are the combined "peak" responses which are used to control fatigue failure.

In accordance with each of these stresses, a design criterion is defined in terms of a designated stress. For Class 1 piping, the designated stress is the stress intensity which is the largest absolute value of the principal stress difference, or equivalently twice of the maximum shear stress. For Class $2 / 3$ piping, it is the largest absolute value of the principal stresses. The design verification is simply performed through comparing the designated stress for all above-mentioned stress categories with the relevant allowable limits.

In order to define the consequence that a given load case may cause in a piping system, loads are grouped 
into the following five load sets: Design condition and so-called Service limits of Level A, B, C and D. The safety, or the failure and damage, consideration is introduced through defining various design criteria and rules for each of these load sets. In such a way, stress states for loads in Design and Service limit Level A are under the lowest allowable limits, thus ensuring the best normal operation condition in a piping system. Meanwhile, stress states for loads in Service limit Level D can be controlled in such a way that the highest level of material damages is allowed, but a catastrophic damage can be prevented, and the power piping system can in a short time be recovered through reparations.

Over recent years, many attempts have been made to find the reasons why the design rules and requirements in ASME BPV III are, in several aspects, considered to be difficult to understand and use. The difficulty is partially caused by the fact that we need to deal with both thermal and dynamic loads, to control both ductile and brittle failure under several safety considerations.

\section{Loads and Load-Combinations}

Design requirements in ASME BPV III are given in a close association with those five load sets for Design and Service limit Levels A, B, C and D. Each of these load-sets are one or several load-combination(s) of various loads, including static loads, such as DP (design pressure) and DWs (dead weights), thermal static (PO) or transient loads (TT) and other dynamic loads, e.g., SSE (safe-shutdown earthquake) and so on. In Table 1, we show an example of how these load sets are specified in Sweden. In the table, GV stands for dynamic loads caused by Global Vibration, WH for dynamic loads caused by water-hammer effects, and SRV1 and SRV7 for dynamic loads induced by opening or closing 1 or 7 safety relief valve(s), respectively.

We note that the dynamic load GV/SRV1 is generally not included according to ASME BPV III, and it appears here due to additional requirements specified in Swedish Design Specifications. Furthermore,
Table 1 A typical example of load-sets given in a Swedish design specification.

\begin{tabular}{ll}
\hline Load-combinations & $\begin{array}{l}\text { Design/Service limit } \\
\text { level }\end{array}$ \\
\hline PD + DW & Design \\
$\mathrm{PD}+\mathrm{DW}+\mathrm{GV} / \mathrm{SRV} 1$ & Design \\
$\mathrm{PO}+\mathrm{DW}+\mathrm{GV} / \mathrm{SRV} 1$ & Level A \\
$\mathrm{PO}+\mathrm{DW}+\mathrm{GV} / \mathrm{SRV7}$ & Level B \\
$\mathrm{PO}+\mathrm{DW}+\mathrm{SRSS}(\mathrm{GV} / \mathrm{SRV7}, \mathrm{WH})$ & Level C \\
$\mathrm{PO}+\mathrm{DW}+\mathrm{SRSS}(\mathrm{GV} / \mathrm{SRV7}, \mathrm{GV} / \mathrm{SSE})$ & Level D \\
\hline
\end{tabular}

dynamic loads caused by global vibration are generally given in terms of response spectra. Hence, dynamic loads can be given either in terms response spectra or time-varying loads.

In ASME BPV III, dynamic loads are classified into reversing and non-reversing types and design criteria are closely given in terms of these two types. It is therefore essential to identify what type of dynamic load is dealt with.

A dynamic load which does not cycle about a mean value is of non-reversing type. A dynamic load $F(t)$ which includes a non-reversing dynamic load, or a non-reversing dynamic load followed by a reversing dynamic load, see Fig. 1, should considered as non-reversing type. The definition of a reversing type is principally the same for Class 1 and 2/3 piping systems. For clarity, we repeat the definition for Class 1 piping below.

Class 1 (NB-3622): Reversing dynamic loads are those loads which cycle about a mean value and include building filtered loads and earthquake loads. A reversing load shall be treated as a non-reversing dynamic load when the number of reversing dynamic cycles, excluding earthquake, exceeds 20.

Remarks:

(1) Allowable stress limits for non-reversing type are generally higher than those for reversing type;

(2) Design criteria for Service limit Level A are the same for both reversing and non-reversing types;

(3) For both reversing and non-reversing dynamic loads in Service limit Level B, fatigue verification must always be conducted. 


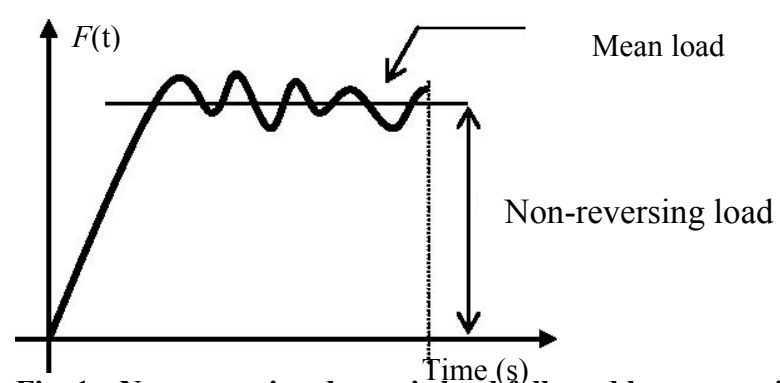

Fig. 1 Non-reversing dynamic load followed by a reversing dynamic load.

\section{Linear and Simplified Elastic-Plastic Fatigue Verification}

The linear design rules for Class 1 piping are given in NB-3653 for Service limit Level A and in NB-3654 for Service limit Level B. Generally, the verifications are required to fulfil two requirements: fatigue and thermal ratchet requirements. Below we shall more specifically address the issue relevant to fatigue verification.

For convenience, notations used in NB-3600 will, unless otherwise stated, be used in this chapter and the following chapters. Moreover, equations cited from NB-3600 will be numbered specifically using the same equation numbers as used in NB-3600, but with a prefixed "NB-". For example, the Eq. (1) in NB-3653.1 will be numbered as "(1)", see Section 4.1. Other equations will, if necessary, be numbered sequentially without repeating those cited equation numbers from NB-3600.

Notice that the fatigue behaviour is entirely caused by a cyclically varying or repeating stress state which is by definition a result of a dynamic event. The fatigue analysis does not, however, specifically deal any transient behaviour in such a dynamic event. The fatigue behaviour for a given material is entirely determined by peak stresses that the material undergoes and the number of load cycles, see Fig. 2. In other word, fatigue damage will never occur without a sufficient number of cyclic changes in stress states no matter what level the maximum stress reaches.

Furthermore, the fatigue verification involves principally following two steps:

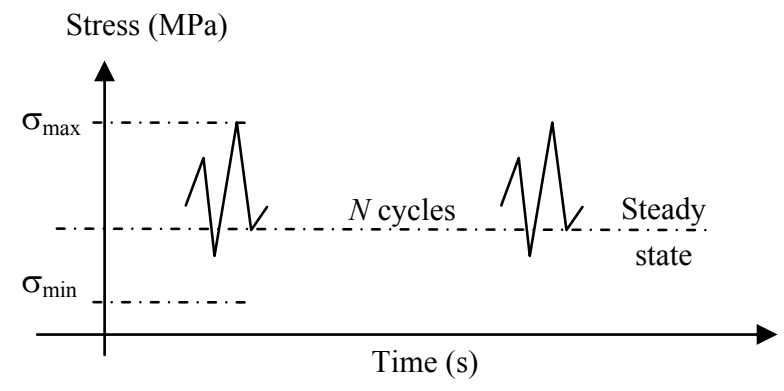

Fig. 2 Cyclically varying/repeating dynamic load.

(1) the maximum total stress range check;

(2) the individual stress range check.

The maximum stress range check must be made for all load sets specified in the Design Specification. Whereas, the later check must be made for all possible pairs of the given load sets, which can be many and, thus, requires a careful book-keeping routine.

\subsection{Linear Verification for Service Limit Level A}

The fatigue requirements that are specified in NB-3653.1-3653.6 are summarized in this section. The verification is often referred as the maximum total stress range check.

(1) Primary plus secondary stress intensity range

The verification is made by requiring a satisfaction of Eq. (1) in NB-3653.1, namely:

$$
\begin{aligned}
s_{n} & =C_{1} \frac{P_{o} D_{o}}{2 t}+C_{2} \frac{D_{o}}{2 I} M_{i}+C_{3} E_{a b} \\
& \times\left|\alpha_{a} T_{a}-\alpha_{b} T_{b}\right| \leq 3 S_{m}
\end{aligned}
$$

to ensure that the elastic shakedown limit is met. We note that the third term in the RHS of Eq. (1) represents the thermal effect due to the gross structural discontinuity. The verification must be conducted for all load sets in Level A.

(2) Peak stress intensity range

The verification is done by using Eq. (2), NB-3653.2, to determine a so-called alternating stress intensity $S_{\text {alt }}$ (NB-3653.3):

$$
\begin{aligned}
s_{p} & =K_{1} C_{1} \frac{P_{o} D_{o}}{2 t}+K_{2} C_{2} \frac{D_{o}}{2 I} M_{i} \\
& +\frac{1}{2(1-v)} K_{3} E \alpha\left|\Delta T_{1}\right| \\
& +K_{3} C_{3} E_{a b} \times\left|\alpha_{a} T_{a}-\alpha_{b} T_{b}\right| \\
& +\frac{1}{1-v} E \alpha\left|\Delta T_{2}\right| \leq 3 S_{m}
\end{aligned}
$$




$$
S_{\text {alt }}=\frac{S_{p}}{2}
$$

This is in turn used to find the allowable number of load cycles $N$ in design fatigue curves (NB-3653.4). Thereafter, a procedure defined in NB-3222.4(e)(5) is applied to estimate the cumulate damage (NB-3653.5), see Box 1 below. The design is qualified if we find a so-called cumulative usage factor $U \leq 1.0$. This verification must be made for all load sets in Level A.

There are many parameters involved in Eqs. (1)-(3), a mong wh i c $\quad \Delta T_{1}, \quad \Delta T_{1} \quad$ and $\alpha_{a} T_{a}-\alpha_{b} T_{b}$ are three so-called temperature gradient parameters which must be determined through a separate, appropriate heat transfer analysis, before making the fatigue verification. In Fig. 3, we illustrate how the absolute values of range of the linear respective non-linear temperature differences through the wall thickness, $\left|\Delta T_{1}\right|$ and $\left|\Delta T_{2}\right|$, can be determined.

The cumulative damage is estimated through a 5-step algorithm given in Box 1 according to NB-3222.4(e)5 for all material points at which fatigue damage is considered to be possible. These material points are mathematically infinitively many. In practice, it is sufficient to select a number of control sections by experiences.

\section{Remarks:}

These fatigue requirements (1) and (2) must also be verified for all load sets which are designated in Service limit Level B, see Section 3.3. When computing the cumulative usage factor $\mathrm{U}$, all load sets in Levels A and B must together be taken into account.

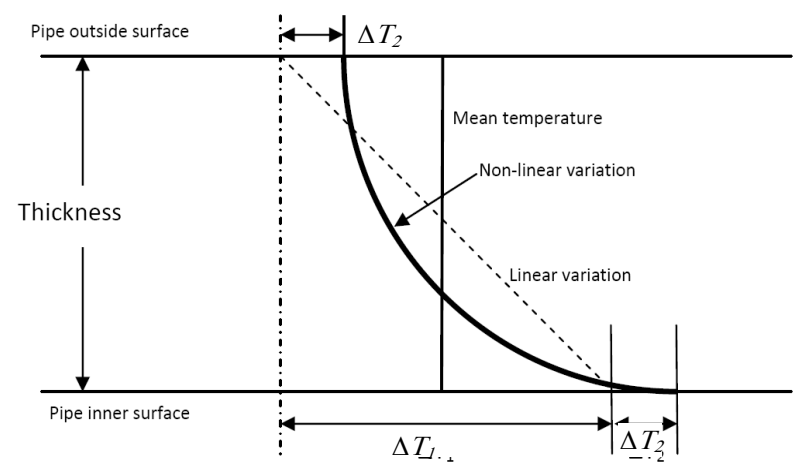

Fig. 3 The linear and non-linear temperature differences through the wall-thickness.
The verification procedure outlined in this section involves entirely linear analyses and, moreover, the cumulative damage usage is determined through an additive assumption, which may explain why this section is entitled as it is.

Box 1 Algorithm for computing the cumulative damage usage factor

Step 1: Let $P_{i}, i=1,2, \ldots, m$, denote $m$ different cyclic loads, and $n_{i}$ the number of load cycles for the load $P_{i}$.

Step 2: Find the alternating stress intensity $S_{\text {alt }(i)}$ for each load $P_{i}$ in accordance with NB-3653.3.

Step 3: Use appropriate design fatigue curves to find for each $S_{a l t(i)}$ the maximum number of load cycles $N_{i}$.

Step 4: Calculate for each cyclic load $P_{\mathrm{i}}$ the fatigue damage usage factor $U_{i}=n_{i} / N_{i}$.

Step 5: Calculate the cumulative damage usage factor by Miner's rule: $U=U_{1}+U_{2}+\ldots+U_{n}$.

4.2 Simplified Elastic-Plastic Verification for Service Limit Level A

In NB-3653.6, an alternative called the simplified elastic-plastic discontinuity analysis is allowed if the linear fatigue verification found to be unsatisfactory. It states that if Eq. (1) unsatisfied, the simplified elastic-plastic discontinuity analysis can be applied to ensure that the plastic shakedown criteria, Eqs. (1)-(3) below, are satisfied.

$$
\begin{aligned}
S_{e} & =C_{2} \frac{D_{o}}{2 I} M_{i}^{*} \leq 3 S_{m} \\
C_{1} & \frac{P_{o} D_{o}}{2 t}+C_{2} \frac{D_{o}}{2 I} M_{i}+C_{3}^{\prime} E_{a b} \\
& \times\left|\alpha_{a} T_{a}-\alpha_{b} T_{b}\right| \leq 3 S_{m}
\end{aligned}
$$

In Eq. (4), the resultant range of moment $M_{i}^{*}$ is the same as that in Eq. (1) except that it includes only moments due to thermal expansion and thermal anchor movements.

Notice that the coefficient $C_{3}^{\prime}$ in Eq. (5) differs from $C_{3}$ in Eq. (1). In Table 2, these coefficients for a few cases are listed. The differences of thermal effect of gross structural discontinuity as considered in the linear and simplified elastic-plastic verification are 
Table 2 Stress indices $C_{3}$ used for linear and simplified elastic-plastic fatigue verification according to Tab. NB-3681-(a)-1.

\begin{tabular}{lll}
\hline & $C_{3}$ & $C_{3}^{\prime}$ \\
\hline Straight pipe & 1.0 & \\
Butt weld reducer & 1.0 & 0.5 \\
Girth fillet weld & 2.0 & 1.0 \\
Butt weld tee & 1.0 & 0.5 \\
\hline
\end{tabular}

clearly shown. It is seen in the table that the thermal effect of gross structural discontinuity is decreased up to $50 \%$ when Eq. (1) is replaced by Eq. (5) when evaluating the primary plus secondary stress intensity range $S_{n}$.

If Eqs. (4) and (5) satisfied, the alternating stress intensity value is calculated by multiplying a modification factor $K_{e}$ to account for the effects of cyclic plasticity by

$$
S_{\text {alt }}=K_{e} \frac{S_{p}}{2}
$$

Here, the peak stress intensity range $S_{p}$ remains unchanged and is determined by Eqs. (2) and (3), the modification factor $K_{e}$, also known as a penalty factor, varies from 1.0 to 5.0 depending on material types and how severely Eq. (5) is violated, i.e., the ratio $S_{n} /\left(3 S_{m}\right)$.

The determination of the penalty factor $K_{e}$ is best illustrated in Fig. 4 below according to NB-3653.6. In the figure, the material parameter $n=0.2$ or 0.3 , see Table NB-3228.5(b1)-1. It is seen in Fig. 4 that, by introducing the penalty factor $K_{e}$, the alternating stress intensity $S_{\text {alt }}$ can be increased by 3.33-5.0 times if Eq. (5) is severely violated, or more precisely, if $S_{n} /\left(3 S_{m}\right) \geq$ 2.0.

Once the alternating stress intensity is determined, the cumulative damage usage factor $U$ is calculated in the same way we do for the linear verification, see Box 1 .

At this point, one may ask: What can we do if Eq. (1) satisfied, but the cumulative damage factor in the requirement (2) found to be $U>1.0$. NB-3600 (and NB-3200) is unclear on this point.

One may realize that, in the simplified elastic-plastic discontinuity analysis, the alternating stress intensity is

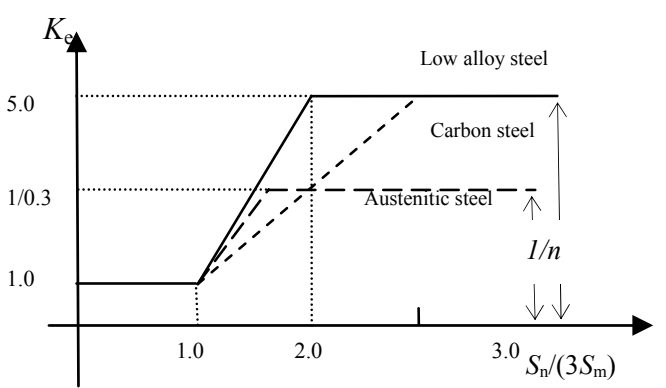

Fig. 4 The penalty factor $K_{e}$ introduced to account for the effects of cyclic plasticity.

increased by a factor $K_{e}=1.0-5.0$ through Eq. (6), which in turn reduces the limit of load-cycles, and consequently increases the cumulative damage factor $U$. In such cases, the simplified elastic-plastic discontinuity analysis will definitively not help in one's further attempt to qualify the piping design.

\subsection{Linear and Simplified Elastic-Plastic Verification for Service Limit Level B}

It is stated in the first paragraph in NB-3654 that the verification procedures for all loads, for which Service limit Level B is designated, are exactly the same as those for Service limit Level A.

However, one may observe immediately, when further examining the requirements specified in NB-3654.2, that the requirements and the required verification procedures are unclear with regard to fatigue requirements. According to NB-3654.2, the verification requirements are basically given in terms of which types of dynamic loads, i.e., non-reversing or reversing, are under consideration. This is not required for Service limit Level A.

The first paragraph in NB-3654 contradicts also with NB-3654.2 regarding how the cumulative damage usage should be computed: Whether all load sets in Levels A and B, or all load sets in Level A and (only) reversing loads in Level $\mathrm{B}$, should together be considered when computing the cumulative damage factor in fatigue verification.

Aiming at bypassing the above difficulties, we agree the following for our currently ongoing power uprate projects: 
(1) For non-reversing loads, or reversing loads combined with non-reversing loads, the requirement given in NB-3654.2(a)) must be fulfilled. That is, we require that Eq. (7) in NB-3652 is satisfied.

$$
B_{1} \frac{P D_{o}}{2 t}+B_{2} \frac{D_{o}}{2 I} M_{i} \leq \min \left(1.8 S_{m}, 1.5 S_{y}\right)
$$

(2) For both reversing and non-reversing dynamic loads, i.e., all loads, the fatigue requirements specified in NB-3653.1 through NB-3653 must be satisfied. In other words, the linear and simplified elastic-plastic fatigue verification procedures outlined above in Sections 4.1 and 4.2 are applied.

We note that the condition (2) above coincides with the first paragraph of NB-3654, but enforces a stronger requirement than that given in NB-3654.2(b) where only reversing dynamic loads are required to fulfil the fatigue requirements.

\section{Remarks:}

The condition (2) is equivalent to designate all loads, which are designated to Service limit Level B, into Service limit level A.

To fulfil the conditions (1) and (2) at the same time implies that a stronger requirement is enforced for loads designated for Level B than those for Level A. Namely, an additional condition (1) is required for non-reversing dynamic loads for Level B. This is obviously not technically reasonable.

Notice from above that the same fatigue requirements are required for Service limit Levels A and $\mathrm{B}$. This is inconsistent, in view of the general design principles upon which ASME BPV III is formulated, see also section. 2 .

\subsection{Accuracy and Reliability of the Simplified Elastic-Plastic Verification}

Among a number of factors that influence on the accuracy and reliability of the simplified elastic-plastic verification, the most notable one is the penalty factor $K_{e}$ induced to account for the effect plastic deformation. We note that the fatigue assessment for nuclear piping and vessels is purely based on strain-controlled fatigue data [7], due to the fact that it is a low-cycle fatigue damage mechanism that we deal with. Stress values in available fatigue design curves are purely pseudo-stresses converted linearly from measured strain values in strain-controlled cyclic tests. Hence, the penalty factor $K_{e}$ is simply an approximation of the ratio of the true strain value $\varepsilon_{\text {true }}$, consisting of both elastic ( $\left.\varepsilon_{\text {true }}^{e}\right)$ and plastic parts $\left(\varepsilon_{\text {true }}^{p}\right)$, to a strain value $\varepsilon_{E}$ computed through a linear elastic analysis. Namely,

$$
K_{e} \approx K_{e}^{\text {true }}=\frac{\varepsilon_{\text {true }}}{\varepsilon_{E}}=\frac{\varepsilon_{\text {true }}^{e}+\varepsilon_{\text {true }}^{p}}{\varepsilon_{E}}
$$

if an additive strain decomposition rule assumed.

It is observed for years that there are many parameters such as geometry, material, load type and so on, which can affect the ratio $K_{e}^{\text {true }}$ and, in addition, the penalty factor $K_{\mathrm{e}}$ suggested in the code, see Fig. 4, can be in many cases far from being an acceptable approximation [8]. Numerical tests for specimens under various load cases conducted recently by Möller [9] reiterate such observations. In his tests, ferritic steel is considered, plan stress conditions are assumed, $\mathrm{J}_{2}$-plasticity with a bilinear kinematic hardening (Plastic modulus $H_{p}=5 \mathrm{GPa}$ ) and without hardening $\left(H_{p}=0\right)$ are used. The tests include a thermal load case for a vessel with its inner surface cyclically heated up and cooled down between $20^{\circ} \mathrm{C}$ and $270{ }^{\circ} \mathrm{C}$. In Fig. 5, the numerical results are summarized and compared to the code. The large discrepancy shown in the figure indicates, though more elaborated verifications needed,

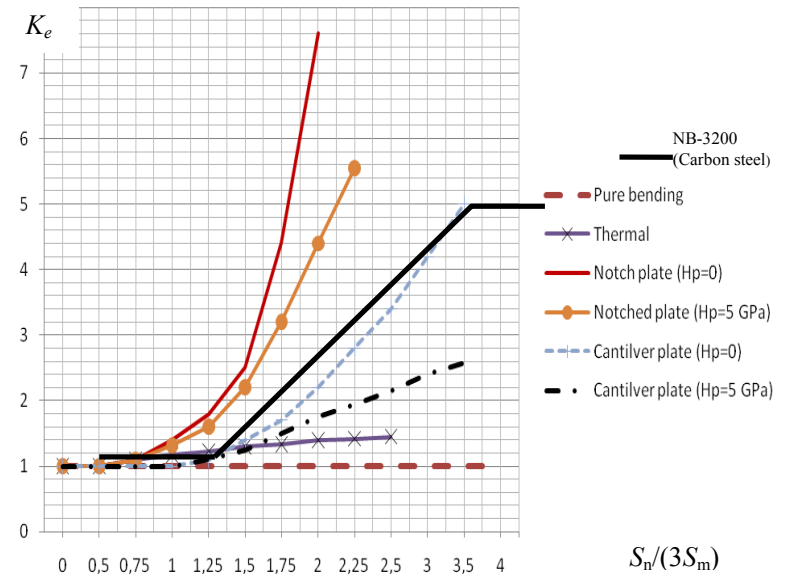

Fig. 5 Large discripency of $K_{e}$ given by NB-3200 as compared to numerical tests. 
that the reliability of the simplified elastic-plastic discontinuous analysis must be questioned seriously.

Indeed, suggestions to correct the penalty factor $K_{e}$ and to correspondingly modify the alternating stress $S_{\text {alt }}$ have been reported, e.g., Slagis [10] and Adams [11] and others. For thermal load cases, an explicit correction based on the idea suggested by Adams is recommended in the code (Code case N-779). In the authors' opinion, this is still far from being a sufficiently reliable and accurate alternative.

\section{Alternative Non-linear Fatigue Verification}

In this chapter, we shall discuss other alternative fatigue verification procedures which are allowed to use if unsatisfactory results found when the linear and simplified elastic-plastic procedures described in Section 4 applied.

\subsection{General}

To begin with, we shall look at NB-3653.1(b). It states that, as an alternative to the simplified elastic-plastic discontinuity analysis in NB-3653.6, one may apply NB-3228.5 Simplified elastic-plastic analysis.

When discussing this issue, we must remind the following: NB-3600 provides design rules and criteria for only piping. Whereas, NB-3200 provides design rules/criteria which are/is more general and detailed and applicable for all nuclear facility components including piping. In other words, NB-3600 provides simplified methods of NB-3200 for performing design-by-analysis for piping. Hence, a piping component which fails to meet conditions in NB-3600 can still be qualified if it meet conditions given in NB-3200. As far as piping concerned, the design rules and requirements given in NB-3200 and NB-3600 should be the same [12].

We now return back to Eq. (1). Recall that Eq. (1) ensures the primary plus secondary stress intensity range being within its limit $3 S_{m}$. By examining NB-3220, we find, however, that none of rules given in NB-3228 seems to be directly applicable for doing a further verification if $U>1$ found in a simplified elastic-plastic analysis. Furthermore, that NB-3200 does not state any further design requirement if the peak stress intensity range leads to a cumulative usage factor $U>1$.

Now, a question arises: Can we apply non-linear analyses to do a further design assessment when Eq. (1), or Eqs. (4) and (5), unsatisfied and/or the cumulative usage factor found to be $U>1$.

Before making attempts to answer the above question, it is worthwhile to briefly examine how thermal ratchet verification is made for Service limit Levels A and B.

\subsection{Thermal Stress Ratchet Verification}

The thermal stress ratchet verification is given in NB-3653.7 which ensures that the range of the linear temperature change through the wall-thickness, $\Delta T_{\text {lrange, }}$, see Fig. 3 , is within a given limit. NB-3653.7 does not state any further assessment rule if the range of temperature changes overshoots its limit. However, in NB-3228.4 Shakedown Analysis, it is stated that a refined non-linear analysis can be used to further examine if the piping components can still be qualified.

In NB-3228.4, both Thermal Stress Ratchet in Shell (NB-3222.5) and Progressive Distortion of Non-integral Connections (NB-3227.3) are discussed. In NB-3228.4(b), it is stated that the design can be considered to be acceptable provided that the following two conditions satisfied:

(1) The maximum accumulated local strain at any point, as a result of cyclic operation to which plastic analysis applied, does not exceed 5\%;

(2) The deformations which occur are within specific limits.

These two conditions can be termed as the $5 \%$ strain limit rule for description convenience.According to NB-3228.4(b), this $5 \%$ strain limit rule is a requirement which replaces the following specific requirements:

(1) NB-3221.2: Local membrane stress intensity being less than $1.5 S_{m}$; 
(2) NB-3222.2: Primary plus secondary stress intensity range being less than $3 S_{m}$, i.e., Eq. (1) in NB-3653;

(3) NB-3222.5: Thermal stress ratchet;

(4) NB-3227.3: Progressive distortion (deformation) control.

In other words, the $5 \%$ strain limit rule sets a limit of progressive deformation in a shakedown process that may eventually take place. We note that this rule applies for both general piping components and non-integral connections (screwed on caps, screwed in plugs, closures etc.).

By thermal stress ratchet it is meant in NB-3222.5 an action, more exactly speaking, a response, in that deformation caused during thermal cyclic loading increases by a nearly equal amount in each cycle. The danger does not lie in the response (deformation) caused in any particular load cycle, but the accumulated amount irreversible (plastic part) response, which may lead to uncontrollable progressive distortion. This may explain why ASME BPV III limits the temperature range $\Delta T_{1 \text { range }}$ in the linear verification, but imposes the $5 \%$ strain limit rule when plasticity considered. For all load sets of Service limit Level A, TT (thermal transients) are of main concern. This implies that a shakedown process is irremissible and the $5 \%$ strain limit rule becomes the right choice.

Now, we consider again the fatigue control or verification. Does this 5\% strain limit rule cover also the need for fatigue control? Generally speaking, it does not. Damage due to fatigue is a totally different damage phenomenon than that caused by material (plastic) yielding. While the former is mostly dominated by brittle failure in form of micro-fracture and cracking, the later is entirely a ductile failure process in which the dislocation of material crystalline grains is dominating. These two damage mechanisms must be dealt with separately.

\subsection{Alternative Fatigue Verification}

Let us return to our question: Can we apply non-linear analyses to do a further design assessment when Eq. (1), or Eqs. (4) and (5), unsatisfied and/or the cumulative usage factor found to be $U>1$ ? For our ongoing power uprate projects, the following procedures are agreed:

If Eqs. (4) and (5) unsatisfied, and $U>1$ (evaluated by the procedure given in NB-3653.6), the 5\% strain limit rule will be applied first. If unsatisfactory, the piping design cannot be qualified and should be replaced.

If the $5 \%$ strain limit rule found to be satisfactory, and a further verification is requested (by the owner of the nuclear power plant), a refined approach for calculating the cumulative usage factor $U$, which is based on the numerical results from non-linear finite element analyses, should be suggested. This should be handled on a base of individual cases. If such an approach agreed, the verification goes further. Otherwise, the design must be declared to be disqualified.

To be able to achieve a more realistic, accurate fatigue assessment, the cumulative usage factor $U$ must be evaluated using refined non-linear finite element solutions in which the effects of cyclic plasticity are considered.

We record again that the fatigue assessment for nuclear piping and vessels is based on strain-controlled fatigue data, due to the fact that it is a low-cycle fatigue damage mechanism that we deal with. In contrast to high-cycle fatigue problems observed in, e.g., aeronautical engineering, where fatigue can be observed principally with little, or without any, plastic deformation taken place, the low-cycle fatigue failure in a few thousand cycles can be produced only by strains in excess of yield strain. Keeping this principle in mind, we can suggest a refined procedure for evaluating the cumulative damage usage factor $U$ in terms of engineering intuition and reasoning.

Notice here that such refined non-linear finite element analyses are already available, which are conducted when attempting to verify the 5\% strain limit rule. The tasks remained are to re-access the 
non-linear finite element solution and extract equivalent elastic and plastic strain solutions at the chosen control sections: the equivalent total peak strain $\varepsilon^{t}$ and its corresponding elastic and plastic parts, $\varepsilon^{e}$ and $\varepsilon^{p}$. From these extracted data, we may find the peak total equivalent strain range $\varepsilon_{\text {range }}^{t}$, and its corresponding elastic and plastic parts, $\varepsilon_{\text {range }}^{e}$ and $\varepsilon_{\text {range }}^{p}$.

We introduce now an alternating equivalent total strain by:

$$
\varepsilon_{\text {alt }}^{e}=\frac{1}{2}\left[\varepsilon_{\text {range }}^{e}+p\left(\varepsilon_{\text {range }}^{p}\right)\right]
$$

where $p\left(\varepsilon_{\text {range }}^{p}\right)$ denotes a polynomial function through which the effects of cyclic plasticity on the fatigue damage can be considered, see discussions below. We note that the elastic part straining $\varepsilon_{\text {range }}^{e}$ will in most cases be much smaller than the plastic part $\varepsilon_{\text {range }}^{p}$.

The alternating stress which accounts for the effects of plastic deformation is simply its elastic counter-part

$$
S_{\text {alt }}=E \varepsilon_{\text {alt }}^{e}
$$

where $E$ is the Young's modulus. The number of allowable cycles $N$ can now be determined using appropriate design fatigue curves. The remained tasks are the same as for the linear verification, see Box 1.

The polynomial function $p\left(\varepsilon_{\text {range }}^{p}\right)$ should be non-linear in order to consistently represent a non-linear fatigue curve. A second-order polynomial may be chosen as follows:

$$
p\left(\varepsilon_{\text {range }}^{p}\right)=a\left(\varepsilon_{\text {range }}^{p}\right)^{2}+b \varepsilon_{\text {range }}^{p}
$$

where $a$ and $b$ are material-dependent parameters which need to be determined through two simple numerical tests using finite element analysis, and fitting to the appropriate fatigue curves.

If we simply choose $a=0$ and $b=1$, the effect of plastic strain range on the alternating stress is linear, and the alternating stress becomes

$$
S_{\text {alt }}=E \varepsilon_{\text {alt }}^{e}=\frac{1}{2} E\left(\varepsilon_{\text {range }}^{e}+\varepsilon_{\text {range }}^{p}\right)=\frac{1}{2} E \varepsilon_{\text {range }}^{t}
$$

Immediately, one can notice that, when the accumulated strain approaches its limit 5\%, the alternating stress will approach to $0.025 E>5,000 \mathrm{MPa}$, which is a huge value and its corresponding allowable number of load cycles will be $N=0$ no matter what fatigue curves are used.

Furthermore, in the simplified elastic-plastic fatigue verification the effect of the cyclic plasticity is accounted by multiplying the (elastic) alternating stress with the penalty factor $K_{e}=1.0 \sim 5.0$. Here, the effect of cyclic plasticity is accounted by adding (partially or totally) the equivalent plastic strain into it elastic counter-part, which equivalently increases the elastic alternating stress. If no plastic deformation occurs, the above-outline procedure becomes identical to the linear procedure. We note the flowing:

(1) The non-linear finite element analysis employed for the verification of the $5 \%$ strain limit rule is a plastic analysis where the true stress-strain relations is used $[2,13,14]$. For our ongoing power uprate projects, such non-linear analyses are made using ANSYS [15];

(2) The analysis must be made for each relevant load set;

(3) When extracting the total strain solutions at a selected control section, it is required to search for two material points, one at the pipe outside surfaces and one at the outside surface, at which the maximum total strain range takes place. For an effective searching, a separate short program which can access the output-database of the finite element analysis is needed.

Moreover, it is only necessary to conduct the re-evaluation of the fatigue usage factor for those control sections at which $U>1$ was found in the earlier verifications.

The above alternative fatigue verification is naturally made in connection with non-linear finite element analysis required for the thermal ratchet verification. Hence, it does not require a large amount of additional work. However, it brings a more reasonable verification than those given in the simplified elastic-plastic verification. The non-linear finite element analysis plays the key role in our alternative fatigue verification, which will be further discussed below. 


\subsection{Non-linear Finite Element Analysis}

Due to practical reasons, non-linear finite element analyses have been made by using $J_{2}$ plasticity without hardening, or with linear or multi-linear hardening assumption for power uprate projects. It has been, however, shown in recent reports by Rahman et al. [16], Hassan et al. [17] and Krishna et al. [18] that such non-linear finite element analyses can only provide a reasonable modeling of plastic shakedown phenomena after a few initial load cycles. For continuous ratcheting responses, such analyses cannot provide reasonable results, neither for the accumulated local strain nor for the global dimension change. They showed through experiments on straight and elbow pipe components that several nonlinear constitutive models available in most general finite element software, such as Chaboche [19], Ohno and Wang [20], and other recently developed models [21-23] can provide a much improved prediction. To achieve a satisfactory modeling, however, they suggested that material parameters involved in these constitutive models need to be calibrated using an inverse or semi-inverse method.

A good prediction of the local accumulated straining is the key for robust fatigue verification. Hence, it is important that more advanced constitutive models must be used in connection with the non-linear finite element analysis on which the alternative fatigue verification.

Among these models, Chaboche non-linear hardening model is available in most general finite element software, such as ANSYS. This hardening model reads as

$$
d a=\sum_{i=1}^{m} d a_{i}=\sum_{i=1}^{m}\left(\frac{2}{3} C_{i} d \varepsilon^{p}-\gamma_{i} a_{i} d p\right)
$$

where $a$ denotes the current center of the yield surface, also known as the back stresses, $\varepsilon^{p}$ the plastic strain tensor and $d p=\left|d \varepsilon^{p}\right|=\left(2 / 3 d \varepsilon^{p} \cdot d \varepsilon^{p}\right)^{1 / 2}$ the magnitude of the plastic strain increment. It is demonstrated that with four decomposed rules $(m=4)$, and corresponding eight material parameters, $C_{i}$ and $\gamma_{i}, i$
$=1,2,3,4$, the model performs reasonably well with small over prediction for most cases. We note that the calibration of these material parameters is not an easy and straight-forward task and requires a fully understanding of cyclic tests and corresponding results.

For other models, numerical implementation may need to be made for interfacing with finite element software. Such an implementation plays en equally important role as the constitutive models themselves for the accuracy and reliability of the ratcheting simulation [24].

\section{Concluding Remarks}

In this paper, fatigue verification of Class 1 nuclear power piping according to ASME BPV III, NB-3600, and relevant issues that are often discussed in connection to power uprate and life-extension of aging BRW reactors in recent years, are addressed. Our emphasis is placed on alternative verification procedures, which do not violate the general design principles upon which ASME BPV III NB-3600 is built, when unaccepted damage is predicted by the simplified elastic-plastic discontinuity analysis. An alternative which employs a non-linear finite element computation and a refined numerical approach for re-evaluating the cumulative damage factors is suggested. This alternative is naturally made in connection with non-linear finite element analysis required for the thermal ratchet verification. Hence, it does not require a large amount of additional work.

General purpose finite element software such as ANSYS and others have become widely available and affordable for large-scale computation with an accuracy and reliability that can never be achieved by other analysis tools. The alternative suggested in this paper is therefore practical. Using this alternative, the plastic deformation which is unavoidable in low-cycle fatigue failure can be correctly taken into account without being affected by uncertainties introduced in the simplified elastic-plastic analysis through the penalty factor $K_{e}$ and other simplifications. It is 
believed that the alternative is the only approach towards an accurate and reliable fatigue assessment.

\section{Acknowledgement}

The work presented in this paper is funded by ÅFORSK, Agreement No. 11-333 and 11-334, which is gratefully acknowledged.

\section{References}

[1] DST Computer Services, S.A., PIPESTRESS User's Manual, Version 3.5.1, Switzerland, June 2004.

[2] Boiler \& Pressure Vessel Code, Section III, Div. 1, Subsections NB and Appendices, The American Society of Mechanical Engineers (ASME), USA, 2010.

[3] L. Zeng, Design Verification of Nuclear Piping According to ASME III and Required Nonlinear Finite Element Analyses, Internal technical report, ÅF-Engineering AB, Sweden, 2007.

[4] L. Zeng, L.G. Jansson, Non-linear design verification of nuclear power piping according to ASME III NB/NC, in: Proc. of 16th Int. Conf. Nuclear Eng. (ICONE16), Orlando, USA, 2008.

[5] L. Zeng, L.G. Jansson, L. Dahlström, More on non-linear verification of nuclear power piping according to ASME III NB/NC, in: Proc. of 17th Int. Conf. Nuclear Eng. (ICONE17), Brussels, Belgium, 2009.

[6] L. Zeng, L.G. Jansson, L. Dahlström, Fatigue verification of class 1 nuclear power piping according to ASME BPV Code, J. Energy and Power Eng. 6 (2012) 519-529.

[7] Criteria of the ASME Boiler and Pressure Vessel Code for Design by Analysis, Sections III and VIII, The American Society of Mechanical Engineers (ASME), N.Y., USA, 1969.

[8] R. Gurdal, S.X. Xu, A comparative study of $K_{e}$-factor in design by analysis for fatigue evaluation, in: Proc. ASME PVP Division Conference, Chicago, USA, 2008.

[9] M. Möller, On the precision of the simplified elastoplastic route in low-cycle fatigue analysis, in: Nordic Symposium on Nuclear Technology 2010, Stockholm, 2010.

[10] G.S. Slagis, Proposed $K_{e}$ Code Cases, ASME Committee Correspondence, 2008

[11] S.A. Adams, An Alternative Rules for Simplified Elastic-Plastic Analysis, Working Group Design
Methodology, BC-05-90, ASME Committee Correspondence, 2008.

[12] G.S. Slagis, G.T. Kitz, Commentary on class 1 piping rules, pressure vessels, piping and components-design and analysis, in: Proc. ASME PVP 1986, 1986.

[13] K.J. Bathe, Finite Element Procedures, Prentice Hall, Englewood Cliffs, NJ, USA, 1996.

[14] M.A. Crisfield, Non-linear Finite Element Analysis of Solids and Structures, Vol. 1, Essentials, Wiley Professional, UK, 1996.

[15] ANSYS, Inc., ANSYS Mechanical-Users' Manual (Version 13), USA, 2013.

[16] S.M. Rahman, T. Hanssan, E. Corona, Evaluation of cyclic plasticity models in ratcheting simulation of straight pipes under cyclic bending and steady internal pressure, Int. J. Plasticity 24 (2008) 1756-1791.

[17] T. Hassan, L. Taleb, S. Krishna, Influence of non-proportional loading on ratcheting response and simulations by two recent cyclic plasticity models, Int. J. Plasticity 24 (2008) 1863-1889.

[18] S. Krishna, T. Hassan, I.B. Naceur, K. Sai, G. Cailletaud, Macro versus micro-scale constitute models in simulating proportaional and non-proportional cyclic and ratcheting responses of stainless steel 304, Int. J. Plasticity 25 (2009) 1910-1949.

[19] J.L. Chaboche, Time independent constitutive theories for cyclic plasticity, Int. J. Plasticity 2 (1986) 149-188.

[20] N. Ohno, J.D. Wang, Kinematic hardening rules with critical state of dynamic recovery-Part I: Formulation and basic features for ratcheting behavior, Int. J. Plasticity 9 (1993) 375-390.

[21] K.M. Abel, N. Ohno, Kinematic hardening model suitable for ratcheting with steady state, Int. J. Plasticity 16 (2000) 225-240.

[22] S. Bari, T. Hassan, An advancement in cyclic plasticity modeling for multiaxial ratcheting simulation, Int. J. Plasticity 18 (2002) 873-894.

[23] X. Chen, R. Jiao, Modified kinematic hardening rule for multiaxial ratcheting prediction, Int. J. Plasticity 20 (2003) 871-898.

[24] L. Zeng, G. Horrigmoe, R. Andersen, Numerical implementation of constitutive integration of rate-independent plasticity, Int. J. Comput. Mech. 18 (5) (1996) 387-396. 